\title{
Teaching Astronomy in Other Cultures: Archeoastronomy
}

\author{
Julieta Fierro \\ Universidad Nacional Autonoma de Mexico \\ E-mail: fierroju@servidor.unam.mx
}

\section{Introduction}

Mesoamerica is a large zone that includes most of Mexico and Central America. In that region one of the few written languages was invented. A large civilization flourished thanks to the discovery of agriculture and the use of a mixture of corn, beans, squash and peppers that conveys a high nutritional content. In this part of the world there are mainly two seasons, the dry and rainy periods. The slave driven civilization that developed in this part of the world consequently divided the civil year in two, mainly dedicated to agriculture and construction. A good calendar was needed for central planning and commerce.

\section{The Sun}

One of the main deities of ancient Mexico was the Sun. It had several representations, an eagle that drifts through the sky and the "Ollin" which means movement. The eagle is depicted in Mexico's flag and the "Ollin" can clearly be seen in the center of the Aztec Calendar and the ten peso coins. A jaguar was the symbol for night, the spots representing stars. Warriors dressed like eagles and jaguars.

In ancient Mexico the sun's trajectory was well studied. The Mesoamerican people carefully registered the places where the sun rises and sets during the year. At mountain surrounded sites they used the silhouette, or carved vertical slots to mark the exact location of the rising sun. At flat locations they built special constructions to register such spots. This way the calendar based on the rising and setting sun was established throughout the region. Mesoamericans also had a 260 day ritual calendar, that ran parallel to the astronomical one.

Some of the markers are for special events such as the equinoxes are for the ball game courts. This game was played with a large rubber ball using the hip to make it go through a stone loop. The days of the equinoxes the sun sets precisely in the direction of the ball court, and can be seen through the stone loop.

There were other constructions built to mark this day such as the "Castle" at Chichen-Itza where the sun forms shadows showing the descent of a snake along the pyramid's stairs. The teacher can bring a souvenir pyramid to the classroom and light it with a lamp in order to simulate the sun's movement and show the way the Maya people could build scale models of their constructions and orient them in a way that they could be useful for astronomical purposes. 
Another marker for the equinox in the Maya region is the "Caracol", an ancient astronomical observatory. The name Caracol means snail; this rounded construction has a spiral shaped hall with several windows that are aligned with particular celestial events such as the rising of Venus on determined dates of calendaric importance.

\section{The Moon}

The Aztecs believed the dark areas of the moon represented a rabbit; they depicted the Moon as a hare in a pot. In fact the Moon's diameter is the same length as Mexico. In Nahuatl, the language of the Aztecs that is still spoken in Mexico, Mexico means "the Moon's navel". Teachers may use the hare image to talk about lunar cratering and volcanism.

Once the moon has been addressed the teacher can explain eclipses. $\mathrm{He} / \mathrm{she}$ can begin by talking about traditions that are still in order since Aztec times. For instance during total eclipses pregnant women tie red ribbons around plants and scissors around their necks "in order to avoid having a deformed child" (in pre-Hispanic times an obsidian arrow head was used instead of scissors). Teachers may explain that the creation of present Mexico City was at the time of a total solar eclipse. Tenochtitlan was founded on a lake where an eagle (the Sun) was resting on a cactus plant (this image is part of the Nation's flag).

The way in which pre-Hispanic people predicted eclipses by carefully observing the Sun's and Moon's trajectories can be explained to students by telling them that since the paths of the sun and moon form a 5 degree angle, and that their apparent motion is different, the moon moves slower, one can infer when the trajectories will cross. It is important to point out the Moon's rising is more erratic that the Sun's and that this jumping of the Moon's motion was another reason for attributing the hare as its deity. It is also an invitation for students to observe its motion.

Part of the rituals included fights amongst eagle and jaguar nights, that I shall recall symbolized the sun and the night,

\section{The Zenith Passing and the Measurement of the Earth's Circum- ference}

One of the most prevailing characteristics in Mesoamerica constructions is perforation drilled in order to observe the zenithal pass of the sun. The ancient calendar began in May with the first rainfall. The zenith pass is during that same period. I shall give a few examples. At Xochicalco there is a cavern built inside one of the pyramids on the main square, where the sun illuminates it during the zenith pass. By the way at this site an astronomical convention was held during the XVth century. The different people from all over Mesoamerica came to decide on calendar matters. This was important for commerce.

At Malinalco, a monolithic pyramid is sculptured from a rock mountain and the sun illuminates a sculpture of an eagle, the sun representation, during the zenith pass. At Monte Albán there is a small construction with a different orientation than the rest of the site, whose staircase points to the pyramid 
dedicated to the zenithal pass. At the same site- and others - there are several gnomons.

A teacher from this area can use this historical fact to show how these sites that are at different latitudes could be used to measure the circumference of the Earth "á la Erathostenes". Using a flat strip of foam rubber the teacher can simulate a flat Earth with obelisks at the same longitude but different latitudes. He can use the same strip to simulate perforations, that is to say zenith passes. Erathostenes used an obelisk and a well; the Mesoamericans could have used perforations. A very good assignment is to use the Internet to have students at different locations measure the circumference of the Earth by observing which day the zenithal path occurs at their location.

\section{Angular measurements}

The ancient people from Mesoamericans were stargazers. Stars were symbolized as eyes. They invented a series of constellations. For instance Orion was represented as a grinding stone, and the Big Dipper by a kite. They used two long rods to measure the altitude of stars above the horizon. The angular distance was determined by pointing one of the rods towards a marker placed on a ceremonial construction and the other at the celestial object.

This fact gives the teacher a chance to address angular measurements on the celestial sphere and to describe the way an astrolabe can be used to determine the altitude of an object above the horizon. A very simple astrolabe can be built using a compass and a weight.

It is difficult to use maps to locate constellations, mainly due to the small scale, that the aspect of the sky varies during the night and year, that not necessarily all the stars shown on the map are in the sky, due to street lights, etc.

In order to avoid these problems the teacher can draw a couple of constellations on rigid plastic sheets with fluorescent paint. Fingernail polish that glows in the dark for discos is ideal. The correct scale is about $30 \mathrm{~cm}$ for Orion. Local as well as the conventional constellations can be used. The teacher should only draw, as many stars as are visible in his particular location. During the night with the arm that is holding the sheet out stretched the size of the constellation should be the same as the one on the drawing.

\section{Conclusion}

Astronomy is a fascinating topic and due to its multidisciplinary nature can be taught in several ways including those that make more sense to a particular group of students. In countries that have great historical tradition it can be employed to help pupils relate to science.

In places such as Mexico, teachers can take the pupils to one of the sites, and explain naked eye observations, and from there build on to modern astrophysics. This will enrich the learning process. 